Background Due to an increased awareness of the significance of genetics in neurodevelopmental disorders (NDD) such as Attention Deficit/Hyperactivity Disorder (ADHD) and autism Spectrum Disorder (ASD), there is an ever-increasing discovery of rare genetic mutations in these patients that are associated risk factors for these NDDs.

$15 \mathrm{q} 11.2$ micro-deletion is a very rare genetic variant where affected patients are likely to require support with learning, have an element of speech delay and/or neuropsychiatric disorders such as ASD and ADHD. It is often due to a de novo mutation but it can also be inherited unknowingly due to variable expressivity.

Genetic chromosomal microarray testing has become increasingly requested for patients with 'unexplained developmental delay/intellectual disability, autism spectrum disorders or multiple congenital anomalies.

Objectives We aimed to describe the clinical, behavioural and learning profile of two patients with $15 \mathrm{q} 11.2$ micro-deletion among the cohort of children and young people (CYP) diagnosed with ADHD in a large Teaching Hospital in the South East of England.

Methods We conducted a search in our hospital electronic database for ADHD patients to identify the occurrence of any associated genetic Copy Number Variants (CNV), specifically looking at the presence of the $15 \mathrm{q} 11.2$ micro-deletion. We also carried out a literature review of the latest research on the association between ADHD and $15 \mathrm{q} 11.2$ micro-deletion using several databases including the OVID, EMBASE, CINHAL and Cochrane's Databases.

Results We identified two ADHD patients with 15q11.2 BP1BP2 micro-deletion, also known as Burnside-Bulter Syndrome. Both of our patients also had learning difficulties with unremarkable neonatal backgrounds. Patient A has additional neuro-developmental features and is currently under assessment for possible ASD. He also presents with mild facial dysmorphic features, which has been reported in 92 out of 200 individuals.

Patient B was a looked-after child due to neglect in infancy.

The published literature regarding $15 \mathrm{q} 11.2$ micro-deletion showed wide variation in the reported signs and symptoms among the affected individuals. Most of the papers reported small case series and the article with the highest number of reported cases included 200 individuals. There are a number of individuals that do not have any apparent signs or symptoms. The most commonly reported features include neurological dysfunction, developmental delay (especially with speech and language and motor delay) and neuropsychiatric disorders such as Autism spectrum disorder and Attention deficit hyperactivity disorder. De novo mutations are reported to account for approximately $5 \%$ to $22 \%$ of all $15 \mathrm{q} 11.2$ micro-deletions. There is also a phenotypic overlap with both Prader-Willi and Angelman syndromes.

Conclusions $15 \mathrm{q} 11.2$ micro-deletion is proving to be one of the 'most common cytogenetic abnormalities' in patients with neurodevelopmental disorders and intellectual impairment. More data would be required to investigate its true significance and value in microarray analysis for all patients referred for ADHD and/or ASD assessment.

Clinicians should consider routine assessment for CNVs in CYP with ADHD, especially if they have other multiple comorbid neurodevelopmental disorders and or dysmorphism.

\section{AN AUDIT OF OUR ADHD SERVICE TO REVIEW ASSESSMENTS BEFORE AND AFTER STARTING ADHD MEDICATION AND TO CHECK COMPLIANCE WITH NICE GUIDELINES}

Chinnaiah Yemula, Kathryn Cope. Cambridgeshire Community Services NHS Trust

\subsection{6/archdischild-2021-rcpch.863}

Background Attention Deficit and Hyperactivity Disorder (ADHD) is a common neurodevelopmental condition seen in $5 \%$ of school age children. Children who are commenced on ADHD medication require regular assessments to ensure safety and continuation of medication and to check progress of their condition.

Objectives We would like to ascertain whether:

- baseline cardiovascular assessment and measurements (height, weight, blood pressure and heart rate) have been carried out on children and young people prior to starting ADHD medication

- the initial assessment and subsequent follow ups met the criteria outlined within NICE guidance (2019)

- there are any safeguarding concerns and if present, escalated appropriately

Methods The sample of children and young people was obtained randomly via SystmOne (electronic record) using the read code 'Attention deficit hyperactivity disorder' to identify them between January to December 2019. They were all started on one of the following medications: Medikinet, Medikinet XL, Matoride XL, Concerta XL, Equasym XL and Elvanse. They were assessed and reviewed by an array of different clinicians.

The audit was reviewed against NICE guidance: Attention Deficit Hyperactivity Disorder: Diagnosis and Management (2019).

Results 20 children and young people were included in the audit. All (100\%) had a baseline height, weight, blood pressure and heart rate $(\mathrm{H}, \mathrm{W}, \mathrm{BP}$ and $\mathrm{HR})$ taken and recorded at their initial assessment. 95\% of the children had the baseline readings of their $\mathrm{H}, \mathrm{W}, \mathrm{BP}$ and $\mathrm{HR}$ documented on their clinic letter, and 75\% had their $\mathrm{BP}$ and $\mathrm{HR}$ percentiles recorded.

As per NICE guidance, children on ADHD medication require a repeat blood $\mathrm{BP}$ and $\mathrm{HR}$ after each dose change and every six months. $25 \%$ of the children in this audit had a repeat $\mathrm{BP}$ and $\mathrm{HR}$ within six months, associated with a medication change or dose adjustment. Similarly, NICE advises that children should have a follow up height every 6 months and a follow up weight every 3 months for children under the age of years and every 6 months for children over the age of 10 years. $20 \%$ of children in this audit had a repeat height and weight within these specified timeframes, and this was also associated with a medication change or dose adjustment.

NICE guidance states that all children should have a cardiovascular assessment prior to starting ADHD medication; this audit found that $85 \%$ of children had documentation pertaining to 'normal heart sounds/heart murmur', and the ADHD pre-drug checklist was completed in $80 \%$ of cases.

Safeguarding was also included in this audit, however only $15 \%$ had current or recent safeguarding needs and none of these required any further escalation. 
Conclusions Undoubtedly some of the results of this audit may have been impacted by the COVID 19 pandemic, which commenced in March 2019 and naturally had a significant impact on service delivery and provision during the months that followed.

With regards to this audit, this is likely to be particularly relevant to the children's blood pressure and heart rate measurements and follow up appointments. To overcome this, Saturday Blood Pressure clinics were established locally and measurements were recorded along with centiles. Any children and young people who were due/overdue these checks were invited to attend to ensure ongoing holistic care. Escalation process is implemented to alert the child's paediatrician in case of concerns and for timely action.

\section{ELEVATED BP AMONG CHILDREN AND ADOLESCENTS WITH ADHD DURING THE INITIAL UK COVID-19 LOCKDOWN: SOCIOECONOMIC EFFECTS AND POSSIBLE INDICATOR OF MENTAL STRESS}

${ }^{1}$ Michael Ogundele, ${ }^{2}$ Hani F Ayyash, ${ }^{3}$ Samuele Cortese. ${ }^{1}$ Bridgewater Community Healthcare NHS Foundation Trust; ${ }^{2}$ Integrated Department of Paediatrics, Mid and South Essex University Hospitals Group, Southend University Hospital NHS Foundation Trust, Westcliff-On-Sea, Essex, UK; ${ }^{3}$ Child and Adolescent Psychiatry, University of Southampton and Solent NHS Trust, UK

\subsection{6/archdischild-2021-rcpch.864}

Background Several non-infectious adverse outcomes have been reported among children and young people (CYP) during the COVID-19 pandemic, including mental health symptoms, even though they are less prone to acute infections. The COVID-19 pandemic lockdown (CPL) was inevitably introduced globally to reduce the risk of infection transmission and necessitated significant restrictions on all social contacts, including school and workplace closures. Chronic stress including social isolation has been hypothesised to constitute a risk factor for hypertension (HT). Therefore, we hypothesised that children and adolescents with ADHD might display higher rates of HT following the CPL.

Objectives We aimed to assess the impact of COVID-19 pandemic lockdown (CPL) on the cardiovascular changes among CYP managed for ADHD within a Community Child Unit.

Methods We carried out a prospective cardiovascular assessment of a cohort of 41 (88\% male) CYP attending routine medical reviews for ADHD treatment in community paediatric clinic in the North-West England within the first 6 weeks of the first wave of the UK-wide Covid-19 lockdown in MarchMay 2020.

Their blood pressures (BP) were measured with regularly calibrated electronic sphygmomanometers based on standard clinical procedures and compared to $\mathrm{BP}$ recorded within the previous one year. Definition of Hypertension (HT) or PreHT was based on the British reference charts for CYP. The CYP were followed up with non-clinic-based BP monitoring at home or by GP. ${ }^{1}$

We assessed the socio-economic status (SES) of each CYP, using the last version of the Index of Multiple Deprivation (IMD) 2019 ranking order of their residential Lower-Layer Super Output Areas, based on the postcodes. Spearman's rank correlation coefficient was used to determine the relationship between the different proportions in a sample in discrete nominal groups. Statistical significance was accepted at the $\mathrm{p}$ value of $<0.05$.

Results Mean age of children studies was 12 years (age range; 5 years 9 months to 18 years), 37/out of 41 (90\%) were on ADHD medications, $92.5 \%$ were on various psycho-stimulant formulations while $7.5 \%$ were on non-stimulants. All the medications were within the lower range of normally approved doses.

There was a statistically significant increase in the HR, diastolic and systolic BP of all the CYP with ADHD during the CPL. There was two- to four-fold increase in the number of ADHD CYP with elevated BP measured after the UK CPL compared to the measurements in the previous 12 months (table 1). This increase in measured BP and HR could not be attributed to any prescribed ADHD medications as the patients had been on the same medications for an average of more than 16 months, all within the lower range of normal doses. There was statistically significant correlation between the duration of the CPL in weeks and the pre-/post-CPL differences between both the systolic and diastolic BP (table 1).

\begin{tabular}{|c|c|c|c|c|c|c|c|}
\hline Wks_Post CPL ${ }^{\$}$ & No-Pts & Avg-HR & $\Delta \mathrm{HR}$ & Avg-SB & Avg- $\Delta S B$ & Avg-DiB & Avg- $\Delta \mathrm{Di}$ \\
\hline 1 & 12 & 84.4 & 1.6 & 116.0 & 15.4 & 73.4 & 14.4 \\
\hline 2 & 11 & 80.6 & 1.9 & 108.5 & 15.2 & 71.5 & 12.9 \\
\hline 3 & 8 & 92.6 & 9 & 110.0 & 5.3 & 75.8 & 11.3 \\
\hline 4 & 9 & 87.7 & 8.2 & 114.2 & 8.7 & 70.3 & 12.1 \\
\hline 5 & 1 & 57 & 9 & 117.0 & -15 & 69 & -9 \\
\hline TOTAL & 41 & 84.8 & 4.8 & 112.7 & 11.2 & 72.5 & 12.3 \\
\hline Rho & & -0.2 & 0.82 & 0.4 & -0.9 & -0.7 & -0.9 \\
\hline$P$ value & & 0.75 & 0.09 & 0.5 & $0.04^{* *}$ & 0.19 & $0.04^{* *}$ \\
\hline
\end{tabular}

\section{Legend:}

Avg - Average (excluding missing readings); No - Number; Pts - ADHD patients; Rho Spearman Coefficient of correlation; CPL - Covid-19 Pandemic lockdown; HR - Heart Rate; SB - Systolic BP; DiB - Diastolic BP

\$ Week 1 starting from 26th March 2020; ** Statistically significant; $\Delta$ Difference between pre- and post-CPL measurements.

73 percent (30/41) of the CYP lived in the most deprived Quintile of the community while 7\% (3/41) lived in the most affluent Quintile. There was no statistically significant difference between the different areas of socio-economic deprivation and the observed changes in the CVS status of the CYP.

Conclusions The pandemic lockdown was associated with statistically significant increase in the $\mathrm{HR}$, diastolic and systolic $\mathrm{BP}$ of all the CYP with ADHD. This finding suggests that there was progressively increasing stress experienced during the lockdown by CYP with ADHD.

It highlights the need of adequate provisions for effective preventive measures to address the significant stress among CYP during future lockdowns or similar emergencies.

This study did not confirm any significant association between different areas of socio-economic deprivation and the observed changes in the CVS status of the CYP.

\section{REFERENCE}

1. Mahmood S et al. Non-pharmacological management of hypertension: in the light of current research. Ir J Med Sci 2019 May;188(2):437-452. 